\title{
Manejo de la obesidad en Atención Primaria
}

\author{
M. MATÉ DEL TÍO, M.D. CANO*, \\ R. Álvarez-Sala Walther**, J. Bilbao Garay*** \\ Especialista en Medicina Interna y Residente de Mediana Familiar y Comunitaria. \\ Hospital "Puerta de Hierro". Madrid, *Especialista en M edicina Familiar y \\ Comunitaria, **M édico Adjunto del Servicio de N eumología. Hospital "La Paz". \\ Madrid, ***J efe de Unidad de Medicina Interna de la Fundación \\ Hospital Alcorcón. Madrid
}

Management of obesity in Primary Health Care

\section{RESUMEN}

La obesidad es uno de los problemas médicos más frecuentes y factor de riesgo para enfermedades como la hipertensión, la diabetes, la artrosis y los eventos cardiovasculares. Es causa de importante morbilidad y mortalidad y genera grandes costes sociales y sanitarios. Además, la obesidad infantil, precede a gran número de complicaciones metabóli cas en la edad adulta. El tratamiento, sobre todo en el síndrome metabólico, debe estar dirigido a la pér. dida de peso. Las más recientes recomendaciones se centran en ayudar a los pacientes a perder un 5$10 \%$ de su peso basal y mantener estas pérdidas a largo plazo. Por esto, escoger el correcto tratamien to es muy importante. En este artículo, se revisan las nuevas recomendaciones sobre la pérdida de peso, y se ofrecen sugerencias para el manejo de estos pa cientes. También se discuten las nuevas opciones de tratamiento y su aplicación en Atención Primaria.

Palabras clave: Obesidad. Complicaciones. Tra tamiento. Prevención.

La obesidad es un trastorno multifactorial caracterizado por un exceso de grasa corporal. Aunque la obesidad ha sido considerada hasta hace poco tiempo, como un exceso de calorías ingerida en relación con las calorías gastadas, cada vez hay más evidencias de los múltiples factores que intervienen en ella. Tampoco ha sido considerada enfermedad como tal, y sin embargo, es el origen de múltiples trastornos sistémicos de importante morbi-mortalidad, que podrían prevenirse o curarse con un buen control del peso. Todo esto, unido a la gran prevalencia, que va en aumento, y a los altos costes sanitarios, ha hecho que pase de ser un problema estético a ser un problema de salud pública mayor ${ }^{1}$.

\begin{abstract}
Obesity is one of the most common medical pro blems and a risk for illnesses such as hypertension, diabetes, degenerative arthritis and cardiovascular events. It is a cause of significant morbidity and mortality and generates great social and financial costs. Morover, childhood obesity precede a great number of metabolic complications in adulthood. The treatment, above all, in the metabolic syndrome, should be weight reduction. Recent weight loss treat ment recommendations have focused on helping $\mathrm{pa}$ tients lose $5-10 \%$ of their body weight and maintai ning these losses for longer durations. Choosing the correct treatment is very important to it. We review the new weight loss recommendations and provide suggestions for assessing patients for weight loss treatment. We also discuss new treatment options and focus on their applications in a Primary Health Care setting.
\end{abstract}

Key words: Obesity. Complications. Treatment. Prevention.

\section{EPIDEMIOLOGÍA}

La Internacional Obesity Task Force (IOTF) resumió recientemente la información disponible sobre la epidemiología de la obesidad, como parte de una consulta de la Organización Mundial de la Salud $(\mathrm{OMS})^{2}$. Este comité clasificó la obesidad en función del índice de masa corporal (IMC) peso (Kg)/talla al cuadrado (metros) y consideró peso saludable un índice de 18,5 a 24,9; sobrepeso, de 25 a 29,9; obesidad clase I, de 30 a 34,9; obesidad clase II, de 35 a 39,9; y obesidad clase III si era igual o más de 40 . Al aplicarse estos valores, la prevalencia de obesidad entre la población europea de edad media es del 
15-20\%. Las cifras resultan algo mejores en Escandinavia y Holanda (alrededor del $10 \%$ ) y peores en Europa del Este (del 40 al 50\%). En España, recientemente se ha publicado un metaanálisis derivado de cuatro estudios previos, realizados en las Comunidades Autónomas de Cataluña, País Vasco, Madrid y Valencia ${ }^{3}$. Se consideró el peso, talla e índice cintura/cadera obtenidos por medición directa en 5.388 individuos entre 25 y 60 años. Se utilizó el IMC como indicador para la tipificación ponderal con un punto de corte $>30$ para definir la obesidad. La prevalencia global fue de $13,4 \%$ (11,5\% en los varones y $15,2 \%$ en las mujeres), pero si se agrupaba por edades, la prevalencia aumentaba con la edad, siendo del $26,3 \%$ en el grupo de 55 a 60 años. Además, el 30,7\% de los varones y el $25,1 \%$ de las mujeres con un IMC> 30 presentaron valores de riesgo para el índice cintura/cadera. El nivel cultural se relacionó inversamente con la prevalencia de la obesidad.

La obesidad infantil tiene especial trascendencia porque muchos niños obesos seguirán siéndolo al convertirse en adultos. Las observaciones indican que la prevalencia de obesidad infantil es elevada, en medios en los que la obesidad es frecuente entre los adultos ${ }^{4}$. Por otro lado, un estudio demostró, tras realizar seguimientos prolongados, que aquellos niños entre 2 y 14 años que estaban por encima del percentil 75 de IMC para su edad, tienen el doble de mortalidad por enfermedad coronaria en la edad adulta tardía que aquéllos que estaban entre el percentil 25 y $50^{5}$. Hay también datos que revelan que en EE.UU. la incidencia de diabetes tipo 2 ha aumentado 10 veces desde 1982 a 1994 entre adolescentes, y se observó que de los casos diagnosticados todos eran obesos $^{6}$.

Desde el punto de vista económico, en diversos países se ha estimado la carga económica asociada a la obesidad ${ }^{7}$. El cálculo incluye no sólo el coste del tratamiento de la obesidad sino también el coste correspondiente a enfermedades atribuibles a ella como hipertensión arterial, dislipemia, gota, diabetes, cáncer y enfermedades cardiovasculares. También se ha estimado el coste de la jubilación anticipada y del desempleo debidos a la obesidad. Se restaron los efectos beneficiosos de la obesidad observados en algunos estudios, como una menor incidencia de fracturas por el menor riesgo de osteoporosis. Con todo esto, una estimación conservadora ha valorado los costes económicos de la obesidad en el $38 \%$ del coste sanitario total en países como Finlandia, Holanda, Francia, EE.UU., Australia y Suecia (porcentaje como mínimo tan importante como el generado por todos los cánceres o por el sida).

\section{FISIOPATOLOGÍA}

La obesidad no es simplemente el resultado de un balance positivo entre la ingestión de calorías y el gasto energético. De forma similar a la hipertensión, la obesidad resulta de la conjunción de múltiples fac- tores ambientales en una persona genéticamente predispuesta ${ }^{8,9}$. Estudios genéticos de obesidad en ratones han conseguido aislar varios genes relacionados. El gen más conocido es el ob, que se expresa en el tejido adiposo. Este gen transcribe una proteína que se secreta llamada leptina. Las mutaciones que causan obesidad en ratones conllevan una disminución en la producción de leptina, o bien, la producción de una leptina no funcional. De forma experimental, se ha visto que la leptina a través de receptores que se expresan en regiones específicas del hipotálamo, actúa de distintas maneras. Por un lado, aumenta el gasto energético y la temperatura corporal, y por otro lado, disminuye el apetito y el peso, tanto en ratones obesos con déficit de leptina como en ratones normales ${ }^{10}$. Este hecho apoya el que exista algún mecanismo feedback entre el tejido adiposo y áreas del sistema nervioso central que regule el apetito y la actividad del metabolismo. Así, algunos trabajos han demostrado la importancia de la leptina en la regulación de algunos ejes neuroendocrinos, como el eje hipotálamo-pituitario-adrenal y el eje hipotálamo-pituitariogonadal $^{11}$. Estudios en humanos han demostrado, que al contrario que en ratones, la obesidad se asocia con un incremento de leptina. Dichos datos sugieren algún tipo de resistencia a la leptina a nivel central ${ }^{12}$. Se ha visto también ${ }^{13}$, que en personas obesas, el gasto energético disminuye porcentualmente más con mayores pérdidas de peso, como si por algún mecanismo de feed-back, el organismo tratara de regresar a su peso original.

La obesidad es un factor de riesgo mayor para la diabetes mellitus (DM) tipo 2, sobre todo si la obesidad es de distribución central o visceral. La DM tipo 2 se caracteriza en las fases iniciales por insulin resistencia e hiperinsulinemia, y en las fases finales por insulin-resistencia e insulinopenia. Es común que se asocie con obesidad, hipertensión y dislipemia (síndrome X). La obesidad se ha asociado con un incremento local y/o sistémico de una serie de hormonas y citoquinas que se sabe inducen insulinresistencia. Las principales son los corticosteroides, los andrógenos y el TNF-alfa. Además, el aumento de triacilgliceroles y ácidos grasos libres (AGL) que se observa en individuos obesos (sobre todo con obesidad central) puede contribuir a la insulin-resistencia e hiperinsulinemia ${ }^{14}$. Otras observaciones que clarifican la fisiopatología de la obesidad y los trastornos relacionados es que: se han tratado animales con obesidad e insulin-resistencia con leptina y se ha obtenido mejoría de ambas anormalidades ${ }^{10}$. Sin embargo, no se sabe si la mejora en la sensibilidad de la insulina es independiente de o secundaria a la pérdida de peso; la sobreexpresión de TNF de tejido adiposo que se observa en obesos disminuye con la pérdida de peso ${ }^{15}$; en animales, el TNF induce la producción de leptina en el tejido adiposo ${ }^{16}$; la insulin-resistencia se asocia con niveles elevados de leptina, independientemente de la grasa corporal ${ }^{17}$; se han identificado receptores de leptina en las células beta pancreáticas ${ }^{18}$. 


\section{DIAGNÓSTICO Y SUBGRUPOS}

Si bien el IMC es una estimación de la masa corporal total, también es importante tener en cuenta la distribución de la grasa. La centralización de la grasa corporal, particularmente en los depósitos intraabdominales o viscerales, se asocia con las complicaciones metabólicas de la obesidad; en cambio, la asociación con estas complicaciones es mucho más débil, cuando el exceso de grasa se concentra en el tejido adiposo glúteo-femoral (Tabla I). También, el síndrome de apnea del sueño (SAOS) se asocia con obesidad de la parte superior del cuerpo, central y sobre todo cervical. Se ha visto que la circunferencia del cuello es el mejor predictor clínico de SAOS, y un estudio ha valorado este dato como medida diagnóstica y ha calculado una sensibilidad del $87 \%$ y una especificidad del $79 \%$, con un valor predictivo positivo del $66 \%$ para SAOS significativo ${ }^{19}$.

En la práctica clínica un índice sencillo que valora la centralización de la grasa corporal, es la relación entre circunferencia de cintura y circunferencia de cadera (RCC). La cintura se mide tras una noche de ayuno en el punto medio entre el arco costal inferior y la cresta ilíaca, mientras que la circunferencia de la cadera se mide en la parte más ancha de la región glútea. La RCC no debería ser superior a 1,0 en varones y a 0,85 en mujeres, límites basados en datos escandinavos ${ }^{20}$.

\begin{tabular}{|l|}
\multicolumn{1}{c|}{ Tabla I } \\
$\begin{array}{c}\text { RIESGO RELATIVO (RR) DE LOS PROBLEMAS DE SALUD } \\
\text { ASOCIADOS A LA OBESIDAD }\end{array}$ \\
\hline MUY AUMENTADO (RR>3) \\
\hline Diabetes \\
Enfermedad de la vesícula biliar \\
Dislipemia \\
Resistencia a la insulina \\
Sensación de falta de aire-síndrome de \\
hipoventilación \\
Síndrome de apnea del sueño \\
MoDERADAMENTE AUMENTADO (RR: 2-3) \\
Enfermedad coronaria \\
Hipertensión arterial \\
Artrosis \\
Hiperuricemia y gota \\
LIGERAMENTE AUMENTADO (RR=1-2) \\
Cáncer (ca. mama en mujeres postmenopáusicas, \\
ca. endometrial, ca. colon) \\
Anormalidades en las hormonas sexuales \\
Síndrome de ovario poliquístico \\
Fertilidad disminuida \\
Aumento de anomalías fetales como consecuencia \\
de la obesidad materna \\
Dolor lumbar \\
Aumento del riesgo anestésico \\
\hline
\end{tabular}

Por tanto, la distribución de la grasa corporal puede evaluarse fácilmente con medidas antropométricas. Otras técnicas que miden la grasa abdominal de forma más segura son la tomografía computarizada y la resonancia, sin embargo, no están justificadas en la práctica clínica por su alta relación coste-beneficio. Otro método más sencillo y asequible que mide la composición corporal es el análisis por impedancia bioeléctrica, sin embargo, este método que parece útil para el seguimiento de personas desnutridas que necesitan ganar peso, no ha demostrado su utilidad en el caso contrario ${ }^{21}$.

\section{PREVENCIÓN Y TRATAMIENTO}

De lo dicho hasta ahora se deduce, que lo más saludable es mantener un IMC adecuado a la edad y tipo constitucional. Sin embargo, debe ser el médico, el que de forma individualizada valore hasta qué punto vale la pena insistir en un cambio del hábito alimentario, costoso tanto para el médico como para el paciente. Para ello habrá que valorar factores de riesgo asociados (Tabla I). Las recomendaciones de la Sociedad Americana de la Obesidad se reflejan en las tablas II y III'22.

Aunque apenas se hayan realizado investigaciones sobre la prevención de la obesidad, existen evidencias indirectas de que ello es posible. Es conocido, que la obesidad en el adulto es difícil de tratar y los programas que ayudan a tratar el sobrepeso en niños tienen más éxito a largo plazo que en adultos. Por tanto, prevenir la obesidad en la juventud puede ser una forma eficaz de prevenir la obesidad en los adultos $^{23}$. Se están realizando ya en España campañas informativas para adquirir hábitos alimentarios saludables desde la infancia y adolescencia, y sería importante tomar medidas que facilitasen en lo posible la realización de ejercicio de forma atractiva y estimulante a nivel de colegios y ayuntamientos.

El tratamiento debe ser multidisciplinar y prolongado en el tiempo por ser un proceso crónico. Lo más eficaz es combinar todos los recursos disponibles, es decir, mejorar los hábitos alimentarios, aumentar la actividad física, apoyo psicológico, tanto para mejorar posibles conflictos que acompañen al exceso de peso como para ayudar a seguir una dieta y tratamiento farmacológico si es necesario. En obesidades mórbidas se puede recurrir también a cirugía. Se ha visto que la pérdida de peso de un $10 \%$, mejora el control glicémico, la tensión arterial y los niveles de colesterol $^{24}$, por lo que la tendencia de los últimos años en el tratamiento de la obesidad, va dirigida a pérdidas de peso moderadas $(5-10 \%$ del peso) pero sostenidas, en contra de las grandes pérdidas de peso que muchas veces son muy difíciles de mantener ${ }^{25}$.

La dieta es la base y el primer paso del tratamiento. Para instaurar una pauta dietética correcta hace falta conocer las costumbres alimentarias del enfermo, la forma de vida, su historia clínica y las posi- 
Tabla II

RIESGO DE SALUD EN FUNCIÓN DEL BMI

\begin{tabular}{lll}
\hline BMI & Riesgo de salud & Si se asocia a otros factores de riesgo \\
\hline$<25$ & mínimo & bajo \\
$25-<27$ & bajo & moderado \\
$27-<30$ & moderado & alto \\
$30-<35$ & alto & muy alto \\
$35-<40$ & muy alto & extremadamente alto \\
$>40$ & extremadamente alto & extremadamente alto \\
\hline
\end{tabular}

Tomada de: Guidance for Treatment of Adult Obesity. Bethesda MD, Shape Up America and the American Obesity Association, 1996.

bles complicaciones añadidas a la obesidad. El interrogatorio debe realizarlo una persona entrenada y debe ser exhaustivo para poder saber el total de calorías que ingiere diariamente, desde si pone azúcar en el café hasta el consumo de alcohol y refrescos, y por supuesto, el número de comidas, dónde come, con quién, entorno familiar, posibles anomalías del comportamiento alimentario (pica, bulimia, hambre nocturna, etc.). Una vez conocemos los hábitos alimentarios, hemos de intentar hacer una dieta personalizada, adecuándola a su forma de vida, nivel socio-económico y posibles complicaciones. Es básica la motivación del paciente para seguir el régimen y el terapeuta debe estimular al enfermo para que siga haciendo la dieta ya que tiende a cansarse. La reducción de la dieta se hará siempre según la ingesta previa. Diversos estudios, diseñados específicamente para examinar la relación entre dieta y obesidad, encuentran una correlación positiva entre ingesta de grasa y obesidad y una correlación negativa entre ingesta de hidratos de carbono y obesidad. Otros estudios de intervención demuestran que se pueden conseguir pérdidas de peso más altas cuando se aconseja seguir una dieta rica en hidratos de carbono y pobre en grasa, que cuando se marca una dieta pobre en grasa o en calorías únicamente ${ }^{26}$. Las dietas hipocalóricas habitualmente utilizadas aportan de 1.000 $\mathrm{kcal}$ a $1.500 \mathrm{kcal} /$ día y se deben realizar bajo estricto control médico. En algunas ocasiones, en obesidades muy severas, se puede recurrir a dietas de muy bajo contenido calórico (400-600 kcal/día), con los hidratos de carbono suficientes para evitar la cetosis, y que incluyen los ácidos grasos esenciales y vitaminas y minerales recomendados. Se debe beber diariamente 1,5 1 de agua, ya que en las dietas hipocalóricas el catabolismo de las proteínas está aumentado, de manera que la eliminación urinaria de nitrógeno también está aumentada, y esto hace que sea necesario mantener una buena diuresis.

El ejercicio por sí solo no hace perder peso, si no se acompaña de una mejora de los hábitos alimentarios. Lo más efectivo sería aumentar la actividad física habitual, como acostumbrarse a subir escaleras, bajar una parada antes del autobús, aparcar el coche algo lejos de nuestro destino, etc. Lo que parece claro es que el ejercicio es fundamental para mantener el peso perdido y para mejorar las complicaciones metabólicas de la obesidad ${ }^{27,28}$. La adhesión del tratamiento a largo plazo depende en gran medida del apoyo psicológico que reciba el paciente. El cambio de los hábitos alimentarios es lo que va a favorecer el mantenimiento del peso perdido y, en muchas ocasiones, la base del éxito radica en el correcto apoyo psicológico. Con terapias de conducta, Epstein et al ${ }^{29}$ analizando los datos de cuatro ensayos controlados a largo plazo, describen que sobre 158 sujetos después de 10 años, el 33,5\% mantuvieron al menos un descenso del $20 \%$ del peso basal, el $42,2 \%$ estuvieron entre el $20 \%$ menos y el $20 \%$ más del peso basal y que el $20,3 \%$ habían incrementado su peso basal un $20 \%$ o más. En cualquier caso, es importante un control a largo plazo, la participación de la familia, dietas sencillas y aplicables, evitar entornos propicios al exceso de ingesta, potenciar actividades físicas del gusto del paciente, reducir conductas sedentarias como la televisión, marcarse metas a corto y largo plazo alcanzables y medibles, con autoevaluaciones en las que el propio paciente valore las causas de éxitos y fracasos, etc. ${ }^{30}$. En los casos en que la obesidad es secundaria a una ingesta alimentaria ansiosa es absolutamente necesaria una modificación conductual.

En cuanto a los fármacos anti-obesidad, podemos dividirlos en:

-Inhibidores del apetito: de acción catecolamí nica: anfetamina, fentermina, fenproporex, dietilpropiona. Son estimulantes del SNC y producen irritabilidad, nerviosismo, euforia, adicción, taquicardia, arritmia,... por lo que existe consenso generalizado de que su uso no está justificado; agonistas de la se rotonina: fenfluramina y dexfenfluramina. Ciertos efectos secundarios graves motivaron la suspensión cautelar de estos medicamentos en todo el mundo en septiembre de $1997^{31,32}$. La fluoxetina y/o sertralina, son antidepresivos de acción serotoninérgica utilizados en pacientes obesos afectados de graves trastornos de la alimentación ${ }^{33}$, sin embargo en el resto de los casos, su uso no está aceptado porque se han comprobado aumentos de peso a partir de los 6 me$\operatorname{ses}^{34,35}$. La sibutramina es un inhibidor específico de la recaptación de serotonina y noradrenalina de clara 
Tabla III

OPCIONES DE TRATAMIENTO EN FUNCIÓN DEL RIESGO DE SALUD

\begin{tabular}{ll}
\hline RIESGO DE SALUD & OPCIÓN DE TRATAMIENTO \\
\hline Mínimo o bajo & $\begin{array}{l}\text { Dieta saludable y/ o moderado } \\
\text { déficit calórico } \\
\text { Aumentar la actividad física } \\
\text { Cambiar ciertos estilos de vida }\end{array}$ \\
Moderado & $\begin{array}{l}\text { Lo previo +dieta baja en } \\
\text { calorías } \\
\text { Lo previotfármacos }\end{array}$ \\
Muy alto & $\begin{array}{l}\text { Lo previo+dieta muy baja en } \\
\text { calorías }\end{array}$ \\
Extremadamente alto & Lo previo+cirugía
\end{tabular}

Tomada de: Guidance for Treatment of Adult Obesity. Bethesda MD, Shape Up America and the American Obesity Association, 1996.

eficacia $^{36}$. Un estudio controlado con placebo con seguimientos de un año demostró que de los pacientes que recibían $15 \mathrm{mg}$ de sibutramina diariamente, el $65 \%$ perdieron $>5 \%$ del peso corporal, comparado al $29 \%$ de los pacientes que recibían placebo; el $39 \%$ del primer grupo perdieron $>10 \%$ del peso corporal, comparado al $8 \%$ del grupo tratado con placebo. Los beneficios de la sibutramina incluyeron además reducciones en los triglicéridos, ácido úrico, colesterol total y LDL colesterol con incrementos en el HDL colesterol. Los efectos adversos observados incluían sequedad de boca, anorexia o aumento del apetito, estreñimiento, insomnio, mareos y náuseas. Aunque en algunos pacientes se observó aumento de la tensión arterial, los cambios fueron de menos de $4 \mathrm{~mm}$ $\mathrm{Hg}$ de media, siendo menos en aquéllos que perdieron más del $5 \%$ de peso. Menos del 1\% de los pacientes tratados tuvieron que abandonar el ensayo por este motivo ${ }^{37}$.

-Fármacos termogénicos: hormonas tiroideas: su uso no está justificado, ya que la mayoría de los pacientes tienen una función tiroidea normal; dini trofrenol: tiene graves efectos secundarios y no se utiliza; cafeína y nicotina: sus efectos como estimulantes del metabolismo son muy pobres y no suelen usarse; simpaticomiméticos: efedrina. Tiene graves efectos indeseables a nivel de SNC y cardiocirculatorio y no está indicado; agonistas betaadrenérgi cos: los estudios realizados en humanos no han sido satisfactorios.

-Inhibidores de la absorción: en la actualidad, está disponible la tetrahidrolipostatina (THL), con resultados positivos en diversos estudios. La THL inhibe la lipasa pancreática y consecuentemente la hidrólisis de triglicéridos y la absorción de lípidos, impidiendo la absorción del $30 \%$ de grasas de la dieta. Entre los efectos indeseables, al parecer poco relevantes, cabe señalar la absorción reducida de vita- minas liposolubles y trastornos gastrointestinales. Uno de los últimos trabajos ${ }^{38}$, incluyeron 688 pacientes en un ensayo randomizado doble-ciego controlado con placebo con un seguimiento de dos años. El primer año, todos los pacientes recibieron dieta hipocalórica. Al año de seguimiento observaron una disminución de peso del 10,2\% (o 10,3 Kg) en el grupo tratado, comparado con el 6,1\% (o 6,1 Kg) en el grupo placebo. En el segundo año, se dividió cada grupo a su vez en dos grupos (THL o placebo) y todos recibieron dieta eucalórica. Los que continuaron con el tratamiento recuperaron peso, previsiblemente por el cambio de dieta, pero la ganancia fue menor que en el grupo que suspendió la medicación. La diferencia entre ambos grupos fue de $2,4 \mathrm{Kg}$. En conclusión, se observaron diferencias significativas, sin embargo, las diferencias de peso entre ambos grupos no fueron muy importantes. A favor de la THL es que se observaron disminuciones en el colesterol total y LDL-colesterol mayores de las esperadas por la sola pérdida de peso. Este efecto, independiente de la pérdida de peso, probablemente fuera reflejo de la habilidad de la THL para impedir de forma específica la absorción de la grasa. En otro ensayo randomizado, doble-ciego realizado en pacientes obesos con diabetes tipo $2^{39}$, se obervaron pérdidas de peso de más del 5 en el $49 \%$ del grupo tratado en comparación con el 23\% del grupo placebo. Mejoró también el perfil glucémico y lipídico en el grupo tratado. Una vez más, insistir en que tendremos que valorar en cada caso la relación coste-beneficio y los riesgos asociados.

En general, una guía práctica para el tratamiento farmacológico de la obesidad puede ser el siguiente: se debe considerar el tratamiento farmacológico a largo plazo en pacientes con BMI igual o mayor de 30 ; la no pérdida de más de $2 \mathrm{Kg}$ después de 4-8 meses debe ser considerado fracaso terapéutico; el fracaso terapéutico nos debe llevar a emplear otro agente en monoterapia o bien una combinación; si después de varios meses de tratamiento no se consigue perder más peso, no quiere decir que el fármaco se haya hecho ineficaz; el fármaco se debe considerar efectivo si se consiguen y mantienen pérdidas de peso del 5$10 \%$ del peso basal; el tratamiento debe continuarse si los beneficios son mayores a los riesgos ${ }^{40,41}$.

De los fármacos antiobesidad hay que tener también en cuenta que algunos tienen efectos beneficiosos con morbilidades asociadas. Así, el inhibidor de la alfaglicosidasa, acarbosa, parece que induce pérdidas de peso $^{38}$. La troglitazona, la primera droga de la clase conocida como thiazolidinedionas, activa un receptor (PPAR) que controla tanto la sensibilidad de la insulina, como la adipogénesis de los fibroblastos. Este fármaco asociado a la insulina consigue reducir la dosis de ésta hasta en un $40 \%$, y parece que no induce significativas ganancias de peso ${ }^{42}$. La THL, como ya se ha comentado, parece que mejora el control glucémico y lipídico en los pacientes con diabetes tipo $2^{39}$ y la acarbosa ${ }^{43}$ y puede mejorar también el perfil lipídico. 
En conclusión, la obesidad está empezando a tenerse en cuenta como problema de salud pública mayor. Es obligación del médico, sobre todo de Atención Primaria y Medicina Interna, tomar conciencia de ello y trasmitirlo al paciente. Se trata de una enfermedad crónica y el adecuado manejo y seguimiento debe ser también crónico. Es también muy importante detectar obesidades infantiles y tratar de prevenirlas. Informar a los padres del riesgo en la edad adulta ayudará a evitar malos hábitos alimentarios.

\section{CORRESPONDENCIA}

Marta Maté del Tío

Avenida de Juan Andrés 15, $7^{\circ}, 6$

28035 Madrid

\section{Bibliografía}

1. Aronne L. Obesity. Med Clin North Am 1998; 82 (1): 161-81.

2. Obesity: preventing and managing the global epidemic: report of a WHO consultation on obesity, Geneva, une 3-5, 1997. Geneva: World Health Organization, 1998.

3. Aranceta J, Pérez Rodrigo C, Serra Majem LL, Ribas L, Quiles Izquierdo J, Vioque, et al. Grupo Colaborativo Español para el Estudio de Obesidad. Prevalencia de obesidad en España: estudio SEEDO 97. Med Clin (Barc) 1998; 111: 441-5.

4. Guillaume M, Lapidus L, Beckers F, Lambert A, Bjorntorp P. Cardiovascular risk factors in children from the Belgian Luxembourg Province: The Belgian Luxembourg Child Study. Am Epidemiol 1996; 144: 867-80.

5. Gunnell D, Frankel S, Nanchahal K, Peters T, Davey Smith G. Childhood obesity and adult cardiovascular mortality. Am Clin Nutr 1998; 67: 1111-8.

6. Pinhas-Hamiel O, Dolan LM, Daniels SR, Standiford D, Khoury PR, Zeitler P. Increased incidence of non-insulindependent diabetes mellitus among adolescents. Pediatr 1996; 128: 608-15.

7. Seidell J, Deerenberg J. Obesity in Europe: prevalence and consequences for the use of medical care. Pharmaco Econ 1994; 5 (1 Suppl): 38S-44S

8. Bouchard C, Prusse L, Leblanc C, Tremblay A, Thériault G. Inheritance of the amount and distribution of human body fat. Int Obesity 1998; 12: 205-15.

9. Cuatrecasas G, Formiguera X, Foz M. Avances en la base genética de la obesidad. Med Clin (Barc) 1999; 112: 664-8.

10. Muzzin P, Eisensmith RC, Copeland KC, Woo SLC. Correction of obesity and diabetes in genetically obese mice by leptin gene therapy. Proceedings of the National Academy o Sciences USA 1996; 93: 14804-8.

11. Barash IA, Cheung CC, Weigle DS, Ren H, Kabigting EB, Kuijper L, et al. Leptin is a metabolic signal to the reproductive system. Endocrinology 1996; 137: 3144-7.

12. Considine RV, Sinha MK, Heiman ML, Kriauciunas A, Stephens TW, Nyce MR, et al. Serum immunoreactive-leptin concentrations in normal-weight and obese humans. N Engl Med 1996; 334: 292-5.

13. Leibel RL, Rosenbaum M, Hirsch. Changes in energy expenditure resulting from altered body weight. N Engl Med 1995; 332: 621-8.

14. Prins B. Food, obesity and non-insulin-dependent diabetes: are there molecular links? Proc Nutr Soc 1997; 56 (3): 889-98.

15. Dandona P, Weinstock R, Love J, Thusu K, Aljada A, Wadden T. Elevated tumor necrosis factor alfa in sera of obese patients: Fall with weight loss. Diabetes 1996; 45 (Suppl A): 333.

16. Grunfeld C, Zhao C, Fuller J, Pollock A, Moser A, Friedman J, et al. Endotoxin and cytokines induce expression of leptin, the ob gene product, in hamsters. Clin Invest 1996; 97: 2152-7.

17. Segal KR, Landt M, Klein S. Relationship between insulin sensitivity and plasma leptin concentration in lean and obese men. Diabetes 1996; 45: 988-91.

18. Kieffer T, Heller RS, Habener F. Leptin receptors expressed on pancreatic beta-cells. Biochemical and Biophysical Research Communications 1996; 224: 522-7.
19. Davies RJO, Ali NJ, Stradling JR. Neck cicumference and other clinical features in the diagnosis of the obstructive sleep apnoea syndrome. Thorax 1992; 47: 101-5.

20. Björntorp P. Visceral obesity: a "civilization syndrome". Obes Res 1993; 1: 206-22.

21. Fulcher GR, Farrer M, Walker M, Rodham D, Clayton B, Alberti KM. A comparison of measurements of lean body mass derived by bioelectrical impedance, skinfold thickness and total body potassium. A study in obese and non-obese normal subjects. Scand Lab Invest 1991; 51 (3): 245-53.

22. Guidance for Treatment of Adult Obesity. Bethesda, MD, Shape Up America and the American Obesity Association 1996.

23. Story M. School-based approaches for preventing and treating obesity. Int Obes Relat Metab Disord 1999; 23 (2 Suppl): 435-515.

24. Goldstein D. Beneficial effects of modest weight loss. Int Obes Relat Metab Disord 1992; 16: 397-415.

25. Rosenbaum M, Leibel R, Hirsch J. Obesity. N Engl Med 1997; 337: 396-407.

26. Ortega RM, Andres P. Carbohydrates and obesity. Med Clin (Barc) 1998; 110 (20): 797-801.

27. Hirsch J. Obesity: some heat but not enough light. Nature 1997; 387: $27-8$.

28. Hubert HB, Fein Leib M, McNamara PM, Castelli WP. Obesity as an independent risk factor of cardiovascular disease: a 26 year follow-up of participants in the Framingham Heart Study. Circulation 1983; 67: 968-77.

29. Epstein LH, Valoski A, Wing RR, McCurley J. Ten-year outcomes of behavioural, family-based treatment for childhood obesity. Health Psychol 1994; 13: 373-83.

30. Robinson TN. Behavioural treatment of chilhood and adolescent obesity. Int Obes Relat Metab Disord 1999; 23 (2 Suppl): 52S-7S.

31. Abenhaim L, Moride V, Brenot F, Rich S, Benichow J, Kurz X, et al. Appetite suppressant drugs and the risk of primary pulmonary hypertension. N Engl Med 1996; 335: 609-16.

32. Connoly HM, Crary L, McGoon MD, Hensrud DD, Edwards BS, Edwards WD, et al. Valvular heart disease associated with fenfluramine- phentermine. N Engl Med 1997; 337: 581-8.

33. Mayer LE, Walsh BT. The use of selective serotonin reuptake inhibitors in eating disorders. Clin Psychiatry 1998; 59 (15 Suppl): 28S-34S

34. Goldstein D, Rampey AH, Enas GG, Potvin H, Fuldzinski LA, Levine LR. Fluoxetine: a randomized clinical trial in the treatment of obesity. Int Obes 1994; 18: 129-35.

35. Long-term pharmacotherapy in the management of obesity. National Task Force on the prevention and treatment of obesity. JAMA 1996; 276: 1907-15.

36. Hanotin C, Thomas F, Jones SP, Leutenegger E, Drovin P. Efficacy and tolerability of sibutramine in obese patients: a doseranging study. Int Obes 1998; 22: 32-8.

37. Jones SP, Smith IG, Kelly F, Gray A. Long-term weight loss with sibutramine (Abstract). Int Obes 1995; 19 (2 Suppl): 41S.

38. Sjontrom L, Rissanen A, Andersen T, Boldrin M, Golay A, Koppeschaar H, et al. Randomised placebo-controlled trial of orlistat for weight loss and prevention of weight regain in obese patients. Lancet 1998; 352: 167-73. 
39. Hollander PA, Elbein SC, Hirsch IB, Kelley D, McGill J, Taylor T, et al. Role of orlistat in the treatment of obese patients with type 2 diabetes. A 1-year randomized double-blind study. Diabetes Care 1998; 21: 1288-94.

40. Scheen A, Lefebvre P. Pharmacological treatment of obesity: present status. Int Obes Relat Metab Disord 1999; 23 (1 Suppl): 47-53.

41. C-Soriguer F, Tinahones F. Principio de precaución y tratamiento con fármacos de las personas obesas. Med Clin (Barc)
1999; 112 (13): 503-7.

42. Kumar S, Boulton AM, Beck-Nielsen H, Berthezene F, Muggeo $\mathrm{M}$, Persson B, et al. Troglitazone, an insulin action enhancer, improves metabolic control in NIDDM patients. Diabetología 1996; 39: 701-9.

43. Balfour A, McTavish D. Acarbosa-an update of its pharmacology and therapeutic use in diabetes mellitus. Drugs 1993; 46: 1025-54. 